NISSUNA UMANA INVESTIGAZIONE SI PUO DIMANDARE VERA SCIENZIA S'ESSA NON PASSA PER LE MATEMATICHE DIMOSTRAZIONI LEONARDO DA VINCI

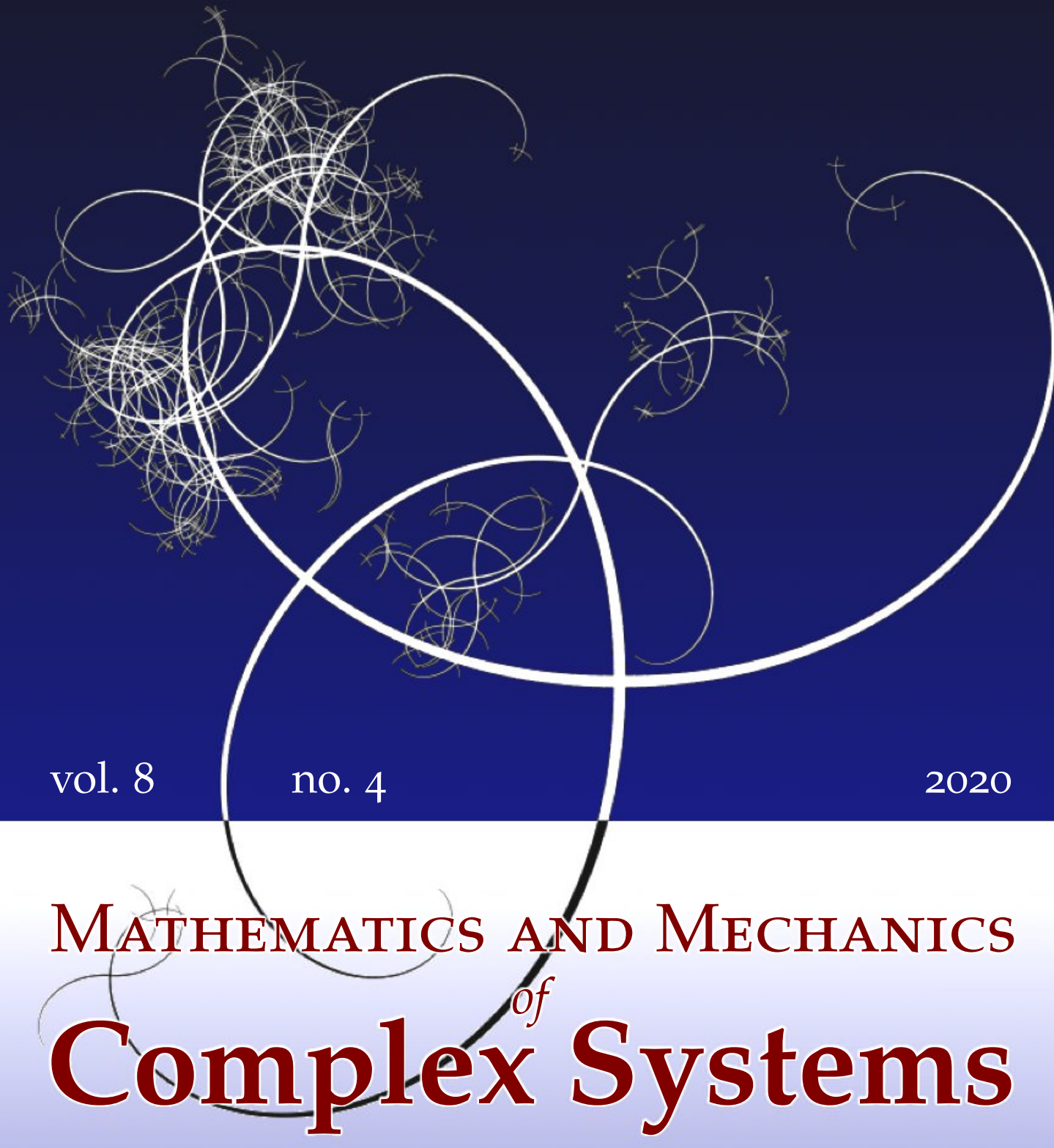

Anna S. Morozova, Elena N. Vilchevskaya, Wolfgang H. MÜller ANd NiKOlay M. Bessonov MODELS FOR DRUG RELEASE OF GENTAMICIN IN A POLYLACTIC ACID MATRIX 


\title{
MODELS FOR DRUG RELEASE OF GENTAMICIN IN A POLYLACTIC ACID MATRIX
}

\author{
Anna S. Morozova, Elena N. Vilchevskaya, \\ WolfGang H. MÜlleR AND NiKOlay M. BESSONOV
}

\begin{abstract}
Recent experiments by Macha et al. (Front. Bioeng. Biotech. 7 (2019), art. id. 37) on the release of gentamicin embedded in a polylactic acid matrix film immersed in a body fluid solution have shown, first, a sudden burst phenomenon after several weeks and, second, a premature end to the release, such that a considerable amount of gentamicin is kept in the matrix. It is shown that such phenomena cannot be described adequately by assuming diffusion of the Fickian kind. In order to improve the modeling, extensions to Fickian diffusion are proposed as follows. The first one is of a phenomenological nature. A production term in the diffusion equation with intrinsic parameters is introduced, all of which can be interpreted intuitively and related to experimental data. The model allows one to capture the aforementioned departure from the timewise parabolic Fickian release characteristic eventually leading to complete release. Second, a micromodel is presented that provides a physical explanation for the proposed production: the drug is released from a carrier particle into the matrix, which eventually comes to an end due to the diminishing particle surface, and the drug adheres to a core due to surface tension. The material parameters of both models are determined by inverse analysis of experimental data.
\end{abstract}

\section{Introduction}

Polylactic acid (PLA) is one of the most common polymers used as a microcarrier of drugs [Jalil and Nixon 1990; Wischke and Schwendeman 2008; Conti et al. 1991]. Low toxicity, excellent biocompatibility, and the absence of inflammation in contact with living organisms, as well as good mechanical properties, make PLA attractive for the pharmaceutical industry, including for the creation of drugretarding systems [Conti et al. 1991].

It is important to predict drug release rates and to understand the primary processes that manage the release. Drug release from biodegradable polymeric carriers, such as a PLA matrix, mostly depends on loading efficiency of the drug,

\section{Communicated by Francesco dell'Isola.}

MSC2020: 60K50.

Keywords: diffusion parameter identification, drug release, diffusion models, finite volumes. 
solubility, biodegradability, diffusion, and the size of the carrier. Mathematical models are an important tool for the development of pharmaceutical compositions, the evaluation of drug release processes, and, in general, the optimal design of new systems [Peppas and Narasimhan 2014].

Significant work has been done on the mathematical modeling of release profiles. See, e.g., [Bruschi 2015, Chapter 5], according to which the most common mathematical models describing drug release are the zero-order model, the first-order model, the Higuchi model, the Hixson and Crowell model, and the KorsmeyerPeppas model.

In the zero-order model the release kinetics of the drug dissolution is only a function of time and the process takes place at a constant rate independent of the active substance concentration. This model holds true only in the case of very slow drug release.

The first-order model is based on the assumption that the change in concentration of drug in the drug carrier with respect to change in time depends only on its concentration and on the phenomenon of dissolution of a solid particle in a liquid with constant rate. The first-order model can be used to describe the drug dissolution in pharmaceutical dosage forms such as those containing soluble active substances incorporated in a porous matrix. For this system the amount of drug released is proportional to the amount of remaining drug in the matrix. Thus, the amount of active release tends to decrease as a function of time.

The Higuchi model [1963] was the first refined mathematical model that described the release of a drug from an insoluble matrix as a square-root-of-timedependent process based on Fickian diffusion. The model was initially derived for planar systems. However, since then it has been modified for use with different geometries and porous systems. This model is based on several assumptions: (i) the drug concentration in the matrix is initially much higher than the solubility of the drug, (ii) edge effects are negligible, so diffusion is unidirectional, (iii) the thickness of the dosage form is much larger than the size of the drug molecules, (iv) the swelling and dissolution of the matrix is negligible, (v) the diffusivity of the drug is constant, and (vi) perfect sink conditions are attained in the release environment.

The Hixson and Crowell model [1931] is used to describe the dissolution process of such dosage forms in which the dissolution surface decreases over time and the geometric shape itself remains the same. For example, this model can be used to describe the dissolution of a specimen consisting of identical spherical particles. This model is based on the assertion that the dissolution rate is proportional to the cube root of weight of the drug particles. When the Hixson and Crowell model is used, it is assumed that the drug release is limited by dissolution velocity and not by diffusion, which can occur through the polymeric matrix. 
The power law model developed in [Korsmeyer et al. 1983; Ritger and Peppas 1987] is a semiempirical equation describing drug release from polymeric systems. This law is useful when the release mechanism is not known or when more than one type of drug release phenomenon is involved. Depending on the value of the exponent of release that best matches the release profile of an active substance in a matrix system, it is possible to establish a classification according to the type of observed behavior: Fickian or non-Fickian models. In the Fickian model the drug release is governed by diffusion. In non-Fickian models the mechanisms driving the drug release are related to the relaxation of polymer chains, diffusion and swelling, or extreme forms of transport. The latter is characterized by tension and breaking of the polymer (solvent crazing).

All of these models can de used to describe the drug release of gentamicin (GM) in PLA carrier [Trang et al. 2019]. However, sudden burst phenomena are observed after several weeks on graphs of GM release from PLA microspheres, which the above models do not take into account. This kind of behavior was also observed in [Macha et al. 2019] during the initial release of GM from a PLA matrix, as well as a premature stop to the release.

Obviously different mathematical models are required to model such observations. To this end we will, in a first step, introduce a phenomenological production term in the classical Fickian diffusion equation, which contains two constitutive parameters for modeling the speed and extend of saturation in the two stages. In a second step, this production term will be introduced and interpreted based on microphysical considerations: the drug is embedded and needs to be delivered to the carrier first. However, the solubility of the embedded drug is limited. The effects resulting from the degradation of the polymer carrier will not be described here but studied in future work instead.

The two developed mathematical models will be tested on the experiment described by Macha et al. [2019], which we briefly summarize in the following passages. It should be noted that they also attempted to fit the data based on simple Fickian diffusion laws. Note that the models presented in this paper go considerably beyond this traditional approach.

In recent experiments by Macha et al. [2019] the dissolution behavior of different drugs in various carriers was investigated. One of the investigated systems concerned the release of GM in a PLA matrix as follows. As a release medium mimicking the body fluid, phosphate-buffered saline solution was used. This solution had a $\mathrm{pH}$ of 7.4 and was kept at a "body temperature" of $37 \pm 0.1^{\circ} \mathrm{C}$. The GM was stored in a nanoporous matrix made of PLA. A scanning electron micrograph (SEM) of the situation is shown in Figure 1. The PLA matrix is shown in gray color whereas GM blisters embedded within the matrix are in white. The distribution of the drug is fairly random. 


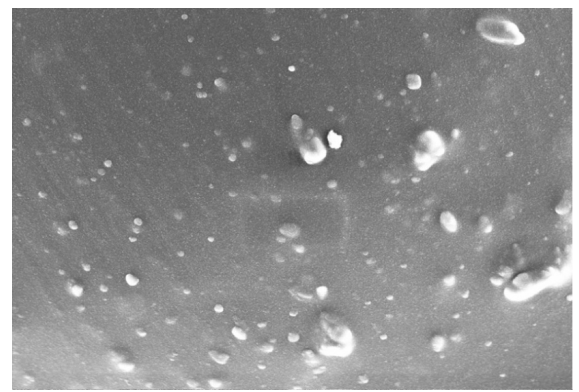

Figure 1. SEM of the GM containing PLA film.

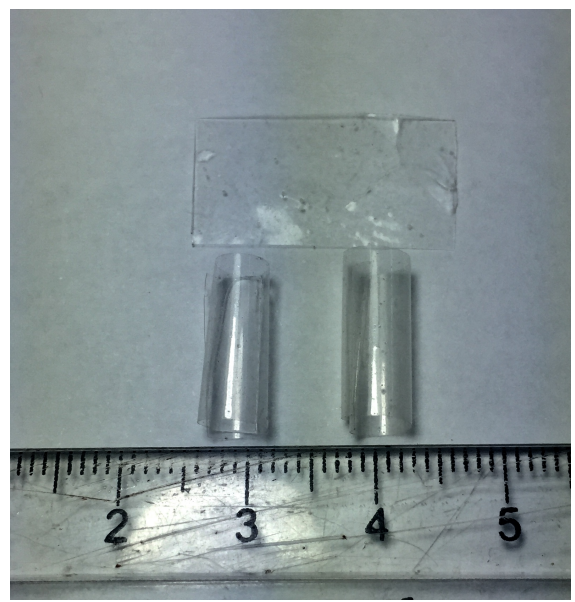

Figure 2. Film matrix specimens before and after curling.

In the experiment the drug-loaded PLA matrix sheet curled up and formed a cylindrical tube. After insertion into a tube filled with solution it slowly started to disintegrate. During this process more and more pore spaces for drug release within the PLA matrix opened up. The film matrix specimens before and after curling are shown in Figure 2.

The initial amount of drug in the matrix was $m_{\mathrm{d}}=7.5 \mathrm{mg}$, and the solution volume was $V_{\mathrm{s}}=15 \mathrm{ml}$. Therefore, the maximum expected drug release concentration is $c_{\max }=0.5 \mathrm{mg} / \mathrm{ml}$. This information was used to calculate the fraction of the drug released at time $t$,

$$
F(t)=\frac{c_{\mathrm{s}}(t)}{c_{\max }}, \quad 0 \leq F(t) \leq 1,
$$

where $c_{\mathrm{s}}(t)=m(t) / V_{\mathrm{s}}$ is the average concentration of the drug in the solution at time $t$. 


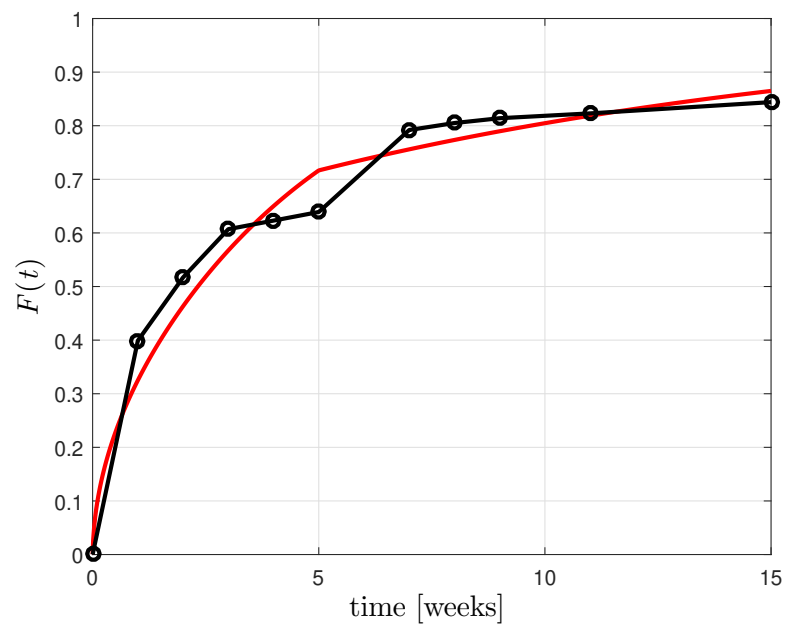

Figure 3. Fractional cumulative release for GM in PLA.

The dependence of the experimentally determined fractional cumulative release on time is indicated by the black circles in Figure 3.

The curve of Figure 3 has several characteristic features. We can clearly distinguish two stages, before and after the fifth week. Moreover, between weeks 1 and 3 the curve runs initially in a parabolic fashion, which indicates Fickian diffusion behavior. However, between weeks 3 and 5 it levels off as if supply of drug is lacking. After that between weeks 5 and 7 we observe another steep boost, which if considered as Fickian would result in a parabolic growth law in time leading to a $100 \%$ dissolution of drug in the body fluid. However, in reality this is not so and again some saturation can be observed. Indeed, the connecting black lines during weeks 5 to 15 clearly indicate a nonparabolic, i.e., non-Fickian behavior.

To demonstrate this quantitatively, an inverse analysis was performed based on a purely Fickian model (similar as in [Macha et al. 2019] or [Rickert et al. 2019], where also more details regarding the computational methods can be found). From the experiment it is known that the matrix curls into a tube. Therefore, we consider a hollow cylinder $\Omega$ of internal and outer radii $r_{\mathrm{i}}=3.2 \mathrm{~mm}$ and $r_{\mathrm{o}}=3.4 \mathrm{~mm}$, respectively, with a height of $H=20 \mathrm{~mm}$; see Figure 4. Since the thickness $d=$ $r_{\mathrm{o}}-r_{\mathrm{i}}=0.2 \mathrm{~mm}$ of the cylinder is very small, it is assumed that the drug is released only through the inner and outer side surfaces $\Gamma$ and not through the rims at the top into the body fluid solution. The distribution in the $z$ direction is assumed to be uniform. In addition it is assumed that compared to the matrix the diffusion of the drug in the body fluid is much faster and a homogeneous distribution is instantaneously assumed. No differential equations need to be used to analyze that process. We assume that all that is released from the matrix into the body fluid 


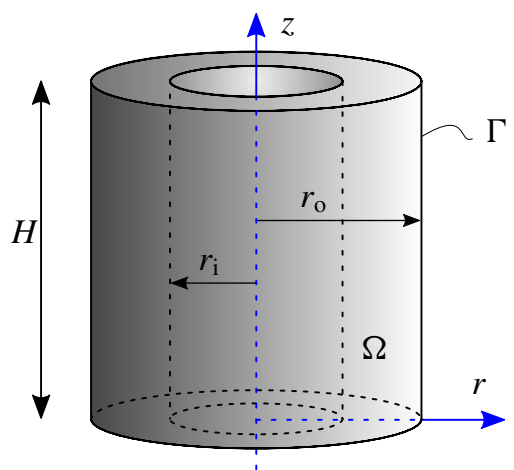

Figure 4. Cylindrical geometry.

is immediately homogeneously distributed in there and contributes to $F(t)$, which can then be calculated by simple algebra. Our goal is to describe the diffusion process within the matrix domain and the mass transfer across the boundary, as well as to determine the diffusion coefficient of the dissolved drug in the matrix, which is one of the key parameters characterizing the kinetics of release. To this end one may think simplistically of using the mass balance together with Fick's first law,

$$
\frac{\partial c(r, t)}{\partial t}=-\nabla \cdot \boldsymbol{J}, \quad \boldsymbol{J}=-D \nabla c(r, t),
$$

in order to obtain the classical diffusion equation, which describes the diffusion process in the matrix $\mathrm{m}$,

$$
\frac{\partial c_{\mathrm{m}}(r, t)}{\partial t}=D \Delta c_{\mathrm{m}}(r, t) \equiv\left(\frac{\partial^{2}}{\partial r^{2}}+\frac{1}{r} \frac{\partial}{\partial r}\right) c_{\mathrm{m}}(r, t) \quad \text { for all } r \in \Omega,
$$

$c_{\mathrm{m}}$ being the concentration of drug in the matrix, which depends on both time and position, and an equation (or boundary/transfer condition) describing the mass transfer across the boundary into the solution $\mathrm{s}$,

$$
V_{\mathrm{s}} \frac{\partial c_{\mathrm{s}}(t)}{\partial t}=\left.2 \pi H D\left(r \frac{\partial c_{\mathrm{m}}(r, t)}{\partial r}\right)\right|_{\Gamma},
$$

where $V_{\mathrm{S}}$ denotes the volume of the solution of body fluid and $c_{\mathrm{S}}(t)$ is the timedependent concentration in the solution.

As initial conditions, a uniform distribution of the drug in the matrix and the absence of the drug in the solution are assumed: $c_{\mathrm{m}}(r, t=0)=c_{0}$ for all $r \in \Omega$ and $c_{\mathrm{S}}(t=0)=0$, where $c_{0}$ is the initial concentration of drug in the matrix,

$$
c_{0}=\frac{m_{\mathrm{d}}}{H \pi\left(r_{\mathrm{o}}^{2}-r_{\mathrm{i}}^{2}\right)} \approx 90.4 \mathrm{mg} / \mathrm{ml} .
$$


The equations of the model were solved numerically by using the finite volume method [Macha et al. 2019; Rickert et al. 2019]. In order to account for the two stages, two corresponding diffusion coefficients were introduced.

The diffusion coefficients for two different stages were determined by minimizing the target function $E=(1 / N) \sum_{i=0}^{N}\left|\left(F_{\exp }\left(t_{i}\right)-F_{\text {num }}\left(t_{i}\right)\right) / F_{\text {num }}\left(t_{i}\right)\right|$, where $F_{\text {exp }}$ refers to the experimentally determined data known at discrete times $t_{i}$ with $i \in[0,10]$. Moreover, $F_{\text {num }}=c_{s}(t) / c_{\max }$ is the numerically predicted function.

The mass release is depicted in Figure 3 by the red curve. The resulting values of the diffusion coefficients for the two stages, (I) $t \in[0,5]$ weeks and (II) $t \in[5,15]$ weeks, are

$$
D_{\mathrm{I}}=1.461 \times 10^{-9} \mathrm{~mm}^{2} / \mathrm{s}, \quad D_{\mathrm{II}}=5.183 \times 10^{-10} \mathrm{~mm}^{2} / \mathrm{s} .
$$

Note that the values differ only slightly from those shown in [Rickert et al. 2019, (12.47)] from a finite element discretization. The following remarks are in order: (i) In stage I the prediction first underestimates the observed release and then begins to take off. There is no leveling off or saturation. (ii) The release prediction in stage II first overshoots a little and then begins to take off. Again it does not allow for a saturation below $100 \%$ of release. It would continue to increase and finally get close to $100 \%$ of cumulative release. (iii) The predicted diffusion coefficient during stage II is less than that of stage I. This is contrary to intuition, because we believe the matrix will start to deteriorate with ongoing time, which creates fissures and, therefore, phenomenologically speaking, the diffusion coefficient should increase. In summary, it is fair to say that this approach is more or less a brute-force fit ignoring possible physical effects based on the simplest type of diffusion equations available. We proceed to improve the situation.

\section{Advanced modeling process}

\subsection{Model 1: a phenomenological production term mimicking a locking effect.}

The main feature of this model is that it allows only a limited precipitation of the drug in the carrier matrix to the solution. It is achieved by adding a phenomenologically introduced sink (or negative production) term $-\chi(r, t)$. Its purpose is to mimic a locking effect in the matrix so that after a certain time $t^{*}$ it will stop the release of the drug. Consequently, we write

$$
\frac{\partial c_{\mathrm{m}}(r, t)}{\partial t}=D \Delta c_{\mathrm{m}}(r, t)-\chi(r, t) \quad \text { for all } r \in \Omega,
$$

while (4), describing the mass transfer across the boundary and the initial conditions, remains unchanged. 

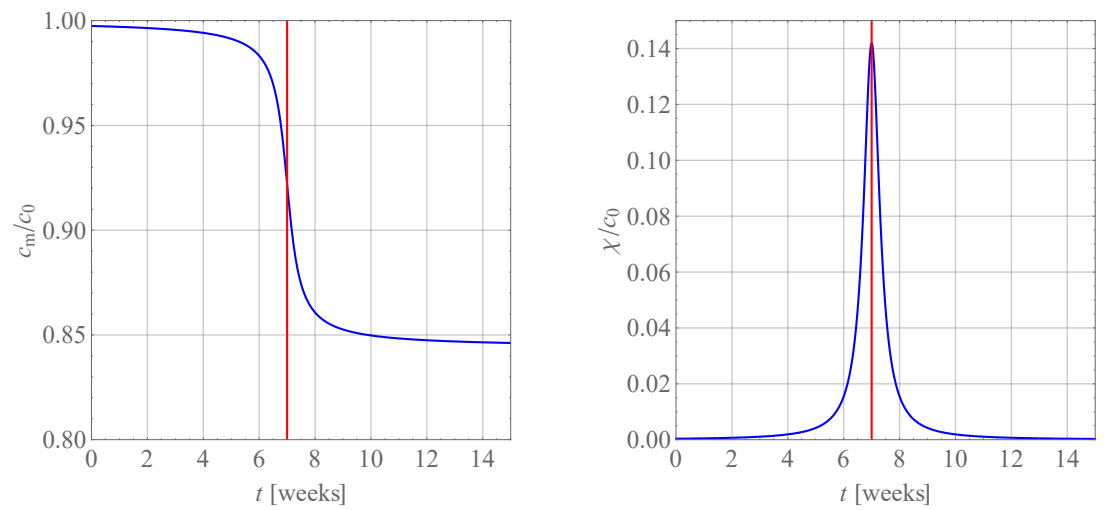

Figure 5. Matrix concentration (9) and production term (11) as a function of time. Time $t_{*}$ is indicated by a red line.

In order to find an explicit expression for $\chi(r, t)$ we first consider (7) without the flux term, i.e.,

$$
\frac{\partial c_{\mathrm{m}}(r, t)}{\partial t}=-\chi(r, t) .
$$

A suitable form of the production is then found by means of a phenomenological argument: we want the concentration of the drug at every point in the matrix to change around a certain point in time, $t_{*}=7$ weeks, from the level $\tilde{c}_{\mathrm{m}}^{*}$, and then finally assumes a constant level $\tilde{c}_{\mathrm{m}}^{\infty}$, where the symbol " $\infty$ " refers to long times, namely 15 weeks. This behavior can be described by using the function

$$
c_{\mathrm{m}}(r, t)=\frac{\tilde{c}_{\mathrm{m}}^{*}-\tilde{c}_{\mathrm{m}}^{\infty}}{\pi} \arctan \left(-\frac{t-t_{*}}{\varepsilon t_{*}}\right)+\frac{\tilde{c}_{\mathrm{m}}^{*}+\tilde{c}_{\mathrm{m}}^{\infty}}{2},
$$

where $\varepsilon$ is a dimensionless parameter allowing us to control the slope of the curve. We can estimate all parameters from the experimental release profile at $t_{*}=7$ and $t_{\infty}=\infty \approx 15$ weeks, i.e., the beginning and the saturation of the second stage:

$$
\tilde{c}_{\mathrm{m}}^{*}=1 \times c_{0}, \quad \tilde{c}_{\mathrm{m}}^{\infty}=0.844 \times c_{0} .
$$

Moreover, in order to guarantee the observed transition sharpness, we choose $\varepsilon=0.05$. The concentration-time function is shown on the left of Figure 5. The transition into saturation is clearly visible.

Then by substituting this function into (8), we obtain the following expression for the production term:

$$
\chi(r, t)=\frac{\tilde{c}_{\mathrm{m}}^{*}-\tilde{c}_{\mathrm{m}}^{\infty}}{\pi \varepsilon t_{*}}\left(1+\left(\frac{t_{*}-t}{\varepsilon t_{*}}\right)^{2}\right)^{-1} .
$$


The production term is shown on the right of Figure 5. As it should be, it is a very sharp function distinctly different from zero only around the time $t_{*}$, which is indicated by the straight red lines in the plots. This also means that there is nearly no production during stage I. Here the release process will essentially be modeled as Fickian.

\subsection{Model 2: production term obtained from a micromodel. Note that Model 1} of Section 2.1 was phenomenological in the sense that we made the drug release stop by prescribing an appropriate production term. In this subsection we attempt to give reasons why the release stops and will "derive" the corresponding production term from micromechanical considerations. To this end, we will assume that initially the matrix as well as the solution do not contain any drug, i.e., $c_{\mathrm{m}}(r, t=0)=0$ for all $r \in \Omega$ and $c_{\mathrm{s}}(t=0)=0$, but rather the matrix will be filled gradually with drug by dissolution of the drug particles stored within. As in (7) of Section 2.1 this process is described by an additional (this time positive) production term $\chi(r, t)$ in the diffusion equation,

$$
\frac{\partial c_{\mathrm{m}}(r, t)}{\partial t}=D \Delta c_{\mathrm{m}}(r, t)+\chi(r, t) \quad \text { for all } r \in \Omega,
$$

and (4) describing the mass transfer across the boundary remains unchanged. The diffusion coefficient $D$ is unknown and will be determined from inverse analysis.

In order to obtain a suitable form for a physics-based production term we now argue microscopically and start from a dissolution law established by Shukarev [Zelikman et al. 1983, p. 424; Wikipedia 2017] to obtain its concrete mathematical form. This law is formulated as follows. The amount of substance transferred across the interface by dissolution of a single particle is proportional to the difference between the concentration at the interface $\left.c\right|_{\widetilde{\Gamma}}$ and the concentration further away, in other words by the "intensity of the sensing phase" $c$, the phase contact surface $\mathrm{d} F$, and time increment $\mathrm{d} t$ :

$$
\mathrm{d} m=\alpha(c \mid \widetilde{\Gamma}-c) \mathrm{d} F \mathrm{~d} t,
$$

where $\mathrm{d} m$ is the mass increment of the solute and $\alpha$ the mass transfer coefficient.

Now in order to get to the continuum scale we homogenize and assume that the drug-carrying particles are all spherical of the same initial radius $R_{0} \approx 7.5 \times 10^{-6} \mathrm{~m}$ based on SEM examinations. For simplicity and in a first step it is also assumed that they are initially uniformly distributed throughout the matrix. This means that the particle density $n=N / V_{\mathrm{m}}$ is constant, while $N$ is the number of particles and $V_{\mathrm{m}}$ is a representative volume element of the matrix. Then Shukarev's law suggests the following form for the production term on the continuum level:

$$
\chi(r, t)=4 \pi R^{2}(t) \alpha(R) n\left[c_{\mathrm{m}} \mid \widetilde{\Gamma}-c_{\mathrm{m}}(r, t)\right] \text { for all } r \in \Omega,
$$


where $R(t)$ is the particle radius, which is a function of time, $n=N / V_{\mathrm{m}}$ is the particle number density, $N$ is the number of particles, $V_{\mathrm{m}}$ is the matrix volume, and $c_{\mathrm{m}} \mid \widetilde{\Gamma}$ is the concentration of dissolved drug at the interface between solid drug and dissolved drug. It will be estimated from the condition that the part of the drug that can dissolve in the remaining volume of the matrix is completely dissolved:

$$
c_{\mathrm{m}} \mid \widetilde{\Gamma}=\frac{N \cdot \rho_{\mathrm{d}} \cdot \frac{4}{3} \pi\left(R_{0}^{3}-R_{\mathrm{cr}}^{3}\right)}{V_{\mathrm{m}}-N \cdot \frac{4}{3} \pi R_{\mathrm{cr}}^{3}},
$$

where $\rho_{\mathrm{d}}=1032 \mathrm{mg} / \mathrm{ml}$ is the mass density of drug and $R_{\mathrm{cr}}$ is a critical radius to which the drug particles dissolve and which will be discussed further. And $\alpha(R)$ is the mass transfer coefficient, which depends on the radius of the drug particle.

We proceed to give a reason why the mass transfer coefficient is not constant but is rather a kinetic characteristic. We assume that it is a function of the (average homogenized) radius $R$ of the drug particles. In fact, the smaller the radius of the particle, the greater the surface tension and therefore the smaller the mass transfer coefficient. We must also take into account that the drug particles do not completely dissolve, i.e., a certain critical radius $R_{\mathrm{cr}}$ exists. When the particle approaches this radius, the production stops, because the stabilizing surface tension is too strong. This fact has been known for a long time [Finholt and Solvang 1968] and observed until today [Dahlberg et al. 2008]. Hence, we propose the following linear approximation carrying all these features:

$$
\alpha(R)=\alpha_{0} \frac{R(t)-R_{\mathrm{cr}}}{R_{0}-R_{\mathrm{cr}}},
$$

where $\alpha_{0}$ is an amplitude to be determined from inverse analysis. As evident by its unit it is characteristic of the speed of dissolution. The critical radius $R_{\mathrm{cr}}$ is a parameter to be adjusted depending on the observed saturation level and can be calculated from mass conservation:

$$
[1-F(15 \text { weeks })] m_{0}=N \cdot \rho_{\mathrm{d}} \cdot \frac{4}{3} \pi R_{\mathrm{cr}}^{3} \Longrightarrow R_{\mathrm{cr}} \approx 0.54 R_{0}, c_{\mathrm{m}} \mid \widetilde{\Gamma} \approx 74 \mathrm{mg} / \mathrm{ml}
$$

In other words, the parameter $R_{\text {cr }}$ puts an end to the dissolution. As mentioned before the idea is that surface tension limits the size of particles that can dissolve. Clearly, the particle radius will change with time until it reaches this limit. Hence, we need a suitable kinetic equation. If we consider the dissolution of one drug particle, Shukarev's law reads

$$
\dot{m}(t)=-\alpha(R)\left[c_{\mathrm{m}} \mid \widetilde{\Gamma}-c_{\mathrm{m}}(r, t)\right] \cdot 4 \pi R^{2}(t) .
$$

We decompose the rate of change of drug mass as a product of drug density and the rate of change in particle volume:

$$
\rho_{\mathrm{d}} \dot{V}(t)=-\alpha(R)\left(c_{\mathrm{m}} \mid \widetilde{\Gamma}-c_{\mathrm{m}}(r, t)\right) \cdot 4 \pi R^{2}(t) .
$$


After substituting the volume of the spherical particle $V(t)=4 / 3 \pi R^{3}(t)$ into (19) we get

$$
\rho_{\mathrm{d}} \frac{\mathrm{d} R(t)}{\mathrm{d} t}=-\alpha(R)\left[c_{\mathrm{m}} \mid \widetilde{\Gamma}-c_{\mathrm{m}}(r, t)\right]
$$

Hence, during the inverse analysis we must solve the coupled system of equations (7), (14), (16), and (20), subject to the initial and boundary conditions mentioned at the beginning of this subsection and $R(0)=R_{0}$.

\section{Results and discussion}

We begin by presenting the results of the phenomenological model with the negative production from Section 2.1. The predicted mass release is shown in Figure 6. Two values for the diffusion coefficients during the two stages were obtained from inverse analysis of the data:

$$
D_{\mathrm{I}}=1.470 \times 10^{-9} \mathrm{~mm}^{2} / \mathrm{s}, \quad D_{\mathrm{II}}=1.509 \times 10^{-9} \mathrm{~mm}^{2} / \mathrm{s} .
$$

Note that $D_{\text {II }}$ is slightly larger than $D_{\text {I }}$. Coincidentally this is confirms our intuition: we expect fissures in the matrix to be generated and to increase during longer exposure of the specimen to the body fluid. Overall this would correspond to an increasing diffusion coefficient. However, it should be emphasized that this result is nothing more than a coincidence and not really substantiated by the model.

Summarizing we may say that the traditional Fickian model of drug release from the matrix and its release into a body fluid solution was extended by introducing a phenomenologically motivated production term. It turns out that the stagnation of the release can be modeled adequately in the second stage during weeks 5 to 15 .

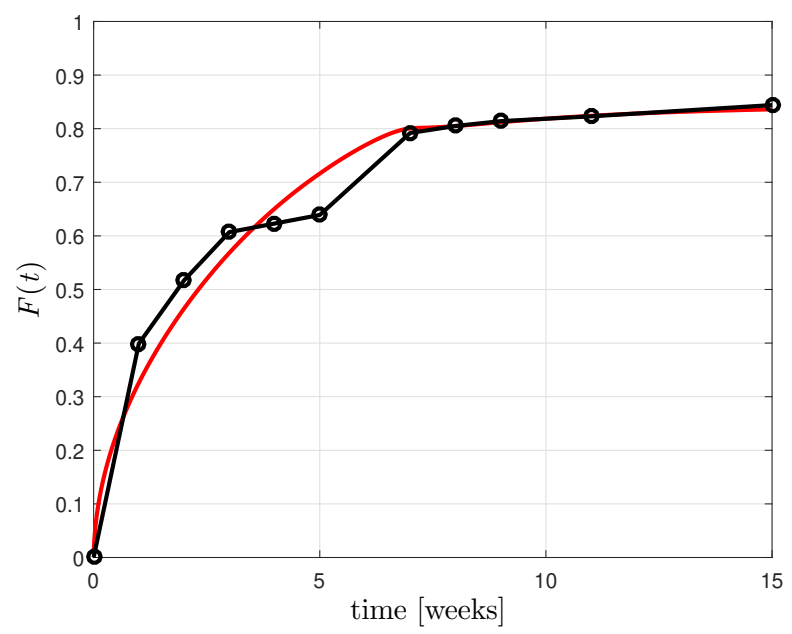

Figure 6. Fractional cumulative release with two-stage diffusion coefficient. 


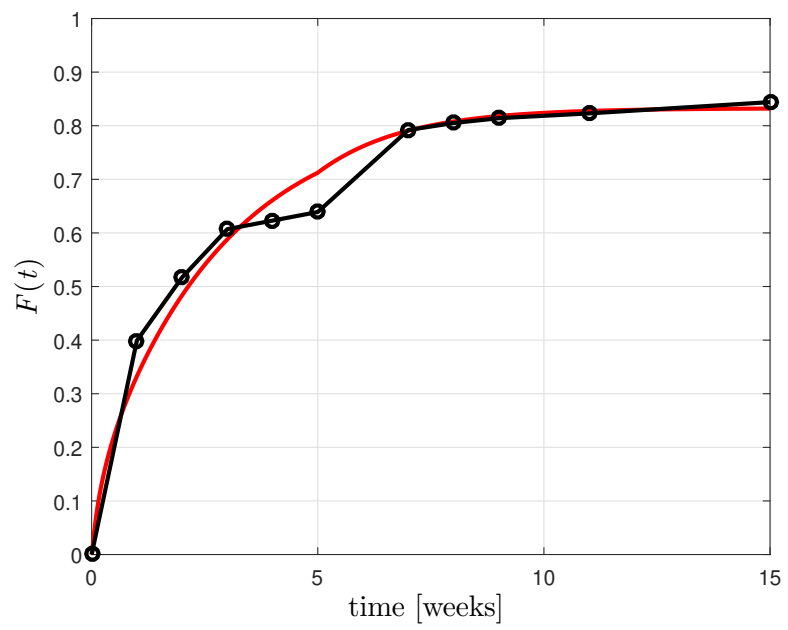

Figure 7. Fractional cumulative release predicted by Shukarev's dissolution model.

We now turn to the micromechanical model with constant particle distribution in the matrix from Section 2.2. The predicted mass release and the experimental data are depicted in Figure 7.

The corresponding values for the diffusion coefficients during the two stages are

$$
D_{\mathrm{I}}=2.418 \times 10^{-9} \mathrm{~mm}^{2} / \mathrm{s}, \quad D_{\mathrm{II}}=3.588 \times 10^{-9} \mathrm{~mm}^{2} / \mathrm{s} .
$$

As part of the inverse analysis the following value for the model parameter of the mass transfer coefficient (16) was obtained: $\alpha_{0}=2 \times 10^{-9} \mathrm{~m} / \mathrm{s}$.

By looking at the graph it turns out that the stagnation of the release can be modeled quite well in the second stage during weeks 5 to 15 . As in the model from Section 2.1, the first stage stagnation after week 3 is not represented at all. Again the diffusion coefficient for the second stage is slightly larger than during the first stage. As discussed before this agrees conveniently with our intuitive view for easier diffusion due to progressing deterioration of the matrix. It is to be understood that the diffusion coefficient is a kinematic characteristic that, generally speaking, depends on many facts (including the ones listed above). Therefore, our assumption about the relationship between the quality of the matrix and the values of the diffusion coefficient has been put into a new perspective.

\section{Conclusions and outlook}

Various mathematical models have been analyzed in order to describe the diffusion process in a carrier matrix and drug release across the boundary. After a short review of results from classical Fickian diffusion an extended diffusion equation 
with two different production terms was presented. These can mimic a locking effect, one on a phenomenological basis and the other one on microscopic grounds regarding the dissolution of individual drug particles.

It is intuitively clear that the deterioration of the quality of the polymer matrix over time can be "incorporated" in the determination of the diffusion coefficient for two different stages - before and after the fifth week.

However, we must conclude that the suggested models can give only an approximate understanding of the drug release described in [Macha et al. 2019], since they do not describe the saturation during the first phase as well as the destruction of the carrier polymer matrix into parts before the active substance is completely released, which is one of the reasons for the burst-type release. The analysis of this is left to future research.

Moreover, our enhanced models may well describe drug dissolution and diffusion in a nonbiodegradable polymer matrix. Such a matrix after the release of the active substance must be removed from the body either mechanically or through the gastrointestinal tract. To describe the release of a drug from such a matrix, we do not need to introduce two stages and two diffusion coefficients.

\section{References}

[Bruschi 2015] M. L. Bruschi, Strategies to modify the drug release from pharmaceutical systems, Woodhead Publishing Series in Biomedicine 85, Woodhead, Cambridge, UK, 2015.

[Conti et al. 1991] B. Conti, F. Pavanetto, and I. Genta, "Use of polylactic acid for the preparation of microparticulate drug delivery systems", J. Microencapsul. 9:2 (1991), 153-166.

[Dahlberg et al. 2008] C. Dahlberg, A. Millqvist-Fureby, and M. Schuleit, "Surface composition and contact angle relationships for differently prepared solid dispersions", Eur. J. Pharm. Biopharm. 70:2 (2008), 478-485.

[Finholt and Solvang 1968] P. Finholt and S. Solvang, "Dissolution kinetics of drugs in human gastric juice: the role of surface tension", J. Pharm. Sci. 57:8 (1968), 1322-1326.

[Higuchi 1963] T. Higuchi, "Mechanism of sustained-action medication: theoretical analysis of rate of release of solid drugs dispersed in solid matrices", J. Pharm. Sci. 52:12 (1963), 1145-1149.

[Hixson and Crowell 1931] A. W. Hixson and J. H. Crowell, "Dependence of reaction velocity upon surface and agitation", Ind. Eng. Chem. 23:8 (1931), 923-931.

[Jalil and Nixon 1990] R. Jalil and J. R. Nixon, "Biodegradable poly(lactic acid) and poly(lactide-coglycolide) microcapsules: problems associated with preparative techniques and release properties", J. Microencapsul. 7:3 (1990), 297-325.

[Korsmeyer et al. 1983] R. W. Korsmeyer, R. Gurny, E. Doelker, P. Buri, and N. A. Peppas, "Mechanisms of solute release from porous hydrophilic polymers", Int. J. Pharm. 15:1 (1983), 25-35.

[Macha et al. 2019] I. J. Macha, B. Ben-Nissan, E. N. Vilchevskaya, A. S. Morozova, B. E. Abali, W. H. Müller, and W. Rickert, "Drug delivery from polymer-based nanopharmaceuticals: an experimental study complemented by simulations of selected diffusion processes", Front. Bioeng. Biotech. 7 (2019), art. id. 37. 
[Peppas and Narasimhan 2014] N. A. Peppas and B. Narasimhan, "Mathematical models in drug delivery: How modeling has shaped the way we design new drug delivery systems", J. Control. Release 190 (2014), 75-81.

[Rickert et al. 2019] W. Rickert, A. Morozova, W. H. Müller, E. N. Vilchevskaya, B. Ben-Nissan, and I. Macha, "Drug delivery from polymer-based nanopharmaceuticals: simulations of selected diffusion processes", Chapter 12, pp. 207-226 in Contributions to advanced dynamics and continuum mechanics, edited by H. Altenbach et al., Advanced Structured Materials 114, Springer, 2019.

[Ritger and Peppas 1987] P. L. Ritger and N. A. Peppas, "A simple equation for description of solute release, I: Fickian and non-Fickian release from non-swellable devices in the form of slabs, spheres, cylinders or discs", J. Control. Release 5:1 (1987), 23-36.

[Trang et al. 2019] T. T. T. Trang, M. Mariatti, H. Y. Badrul, K. Masakazu, X. T. T. Nguyen, and A. A. H. Zuratul, "Drug release profile study of gentamicin encapsulated poly(lactic acid) microspheres for drug delivery", Mater. Today Proc. 17 (2019), 836-845.

[Wikipedia 2017] "Sink condition", Wikipedia entry, 2017, Available at https://en.wikipedia.org/ wiki/Sink_condition?oldid=758830177.

[Wischke and Schwendeman 2008] C. Wischke and S. P. Schwendeman, "Principles of encapsulating hydrophobic drugs in PLA/PLGA microparticles”, Int. J. Pharm. 364:2 (2008), 298-327.

[Zelikman et al. 1983] A. Zelikman, G. Voldman, and L. Belyaevskaya, Theory of hydrometallurgical processes, Metallurgiya, Moscow, 1983. In Russian.

Received 30 Apr 2020. Revised 6 Jul 2020. Accepted 28 Sep 2020.

AnNA S. Morozova: aveeanka@gmail.com

Peter the Great Saint Petersburg Polytechnic University, Saint Petersburg, Russia

ELENA N. VILCHEVSKAYA: vilchevskaya_en@spbstu.ru

Institute for Problems in Mechanical Engineering of the Russian Academy of Sciences,

Saint Petersburg, Russia

WOLFGANG H. MüLLER: wolfgang.h.mueller@tu-berlin.de

Fachgebiet Kontinuumsmechanik und Materialtheorie, Institut für Mechanik,

Technische Universität Berlin, Berlin, Germany

NiKOLAY M. BESSONOV: nickbessonov1@gmail.com

Institute for Problems in Mechanical Engineering of the Russian Academy of Sciences,

Saint Petersburg, Russia 


\section{MATHEMATICS AND MECHANICS OF COMPLEX SYSTEMS}

\author{
EDITORIAL BOARD \\ ANTONIO CARCATERRA \\ ERIC A. CARLEN \\ FRANCESCO DELL'ISOLA \\ RAFFAELE ESPOSITO \\ ALbERT FANNJIANG \\ GILLES A. FRANCFORT \\ Pierangelo Marcati \\ Peter A. Markowich \\ MARTIN OSTOJA-STARZEWSKI \\ PiERRE SEPPECHER \\ David J. STEIGMANN \\ Paul Steinmann \\ PIERRE M. SuQueT \\ MANAGING EDITORS \\ MICOL AMAR \\ EMILIO BARCHIESI \\ MARTIN OsTOJA-STARZEWSKI \\ HONORARY EDITORS \\ TEODOR ATANACKOVIĆ \\ VICTOR BERDICHEVSKY \\ GuY BouchitTÉ \\ FELIX DARVE \\ CARlo Marchioro \\ ERRICO PRESUTTI \\ MARIO PULVIRENTI \\ LuCIO Russo \\ ADVISORY BOARD \\ Holm ALTENBACH \\ HARM ASKES \\ ANDREA BRAIDES \\ MAURO CARFORA \\ ERIC DARVE \\ FABRIZIO DAVÌ \\ ANNA DE MASI \\ Emmanuele DiBenedetto \\ VICTOR A. EREMEYEV \\ BERNOLD FIEDLER \\ IRENE M. GAMBA \\ PIERRE GERMAIN \\ SERgey GaVRiLyuk \\ Timothy J. Healey \\ ROBERT P. LIPTON \\ ANGELO LUONGO \\ JUAN J. MANFREDI \\ JEAN-JACQUES MARIGO \\ ANIL MISRA \\ ROBERTO NATALINI \\ Thomas J. Pence \\ ANDREY PIATNITSKI \\ Miguel A. F. SANJUAN \\ A. P. S. Selvadurai \\ MirosLaV ŠILHAVÝ \\ GEORG STADLER \\ Guido SwEERS \\ LEV TRUSKINOVSKY \\ JUAN J. L. VELÁZQUEZ \\ VINCENZO VESPRI \\ VITALY VOLPERT
}

\section{msp.org/memocs}

Università di Roma "La Sapienza", Italia

Rutgers University, USA

(CO-CHAIR) Università di Roma "La Sapienza", Italia

(TREASURER) Università dell'Aquila, Italia

University of California at Davis, USA

(CO-CHAIR) Université Paris-Nord, France

Università dell'Aquila, Italy

DAMTP Cambridge, UK, and University of Vienna, Austria

(CHAIR MANAGING EDITOR) Univ. of Illinois at Urbana-Champaign, USA

Université du Sud Toulon-Var, France

University of California at Berkeley, USA

Universität Erlangen-Nürnberg, Germany

LMA CNRS Marseille, France

Università di Roma "La Sapienza", Italia

Università degli Studi dell'Aquila, Italy

(CHAIR MANAGING EDITOR) Univ. of Illinois at Urbana-Champaign, USA

University of Novi Sad, Serbia

Wayne State University, USA

Université du Sud Toulon-Var, France

Institut Polytechnique de Grenoble, France

Università di Roma "La Sapienza", Italia

Università di Roma Tor Vergata, Italy

Università di Roma "La Sapienza", Italia

Università di Roma "Tor Vergata", Italia

Otto-von-Guericke-Universität Magdeburg, Germany

University of Sheffield, UK

Università di Roma Tor Vergata, Italia

Università di Pavia, Italia

Stanford University, USA

Università Politecnica delle Marche, Ancona (I), Italy

Università dell'Aquila, Italia

Vanderbilt University, USA

Gdansk University of Technology, Poland

Freie Universität Berlin, Germany

University of Texas at Austin, USA

Courant Institute, New York University, USA

Université Aix-Marseille, France

Cornell University, USA

Louisiana State University, USA

Università dell'Aquila, Italia

University of Pittsburgh, USA

École Polytechnique, France

University of Kansas, USA

Istituto per le Applicazioni del Calcolo "M. Picone", Italy

Michigan State University, USA

Narvik University College, Norway, Russia

Universidad Rey Juan Carlos, Madrid, Spain

McGill University, Canada

Academy of Sciences of the Czech Republic

Courant Institute, New York University, United States

Universität zu Köln, Germany

École Polytechnique, France

Bonn University, Germany

Università di Firenze, Italia

CNRS \& Université Lyon 1, France Angelo Vulpiani \& Università di Roma La Sapienza, Italia

MEMOCS (ISSN 2325-3444 electronic, 2326-7186 printed) is a journal of the International Research Center for the Mathematics and Mechanics of Complex Systems at the Università dell'Aquila, Italy.

Cover image: "Tangle" by @ John Horigan; produced using the Context Free program (contextfreeart.org).

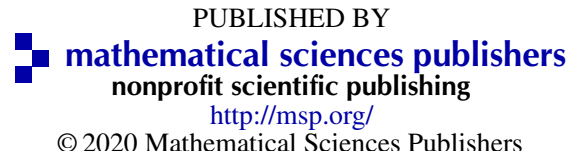

(C) 2020 Mathematical Sciences Publishers 
Mathematics and Mechanics of Complex Systems vol. 8 no. 4

On a stochastic approach to model the double phosphorylation/dephosphorylation cycle

Alberto Maria Bersani, Alessandro Borri, Francesco Carravetta,

Gabriella Mavelli and Pasquale Palumbo

A new comprehensive approach for bone remodeling under medium and high mechanical load based on cellular activity

Daniel George, Rachele Allena, Céline Bourzac, Stéphane Pallu, Morad Bensidhoum, Hugues Portier and Yves Rémond

Models for drug release of gentamicin in a polylactic acid matrix

Anna S. Morozova, Elena N. Vilchevskaya, Wolfgang H. Müller and Nikolay M. Bessonov

Analytical mechanics allows novel vistas on mathematical epidemic dynamics modeling

\section{Paul Steinmann}

A geometrically nonlinear Euler-Bernoulli beam model within strain gradient elasticity with isogeometric analysis and lattice structure applications

Loc V. Tran and Jarkko Niiranen

MEMOCS is a journal of the International Research Center for the Mathematics and Mechanics of Complex Systems at the Università dell' Aquila, Italy.

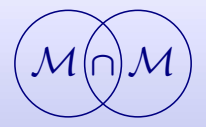

